\title{
ANÁLISE DE GUIAS DE ONDAS CONTENDO MATERIAIS BI- ISOTRÓPICOS PELO MÉTODO FDTD
}

\author{
Licinius Dimitri Sá de Alcantara e Rubem Gonçalves Farias
}

\begin{abstract}
Resumo - Uma formulação numérica baseada no método das Diferenças Finitas no Domínio do Tempo (FDTD) é adaptada visando a simulação da propagação de ondas eletromagnéticas em guias de ondas contendo material biisotrópico. Esta classe de materiais vem focalizando o interesse de pesquisadores nos últimos anos devido às suas propriedades especiais, onde se incluem os materiais quirais, que são caracterizados por criar uma interdependência entre propagação e polarização, trazendo uma perspectiva promissora na difusão de novos dispositivos em telecomunicações.
\end{abstract}

Abstract - A numerical formulation based on the FiniteDifference Time-Domain (FDTD) method is fitted to simulate the electromagnetic waves propagation in waveguides containing bi-isotropic materials. This class of materials has been focusing the researchers interest along the last years due its special properties, especially regarding the chiral materials, which are characterized by generating an interdependence between propagation and polarization that brings a promising perspective concerning the spreading of new telecommunication devices.

Palavras-chaves - Método FDTD, meios bi-isotrópicos, meios quirais.

\section{INTRODUÇÃO}

Materiais bi-isotrópicos. especialmente quirais, vêm gradativamente assumindo uma posição de destaque em pesquisas na área de eletromagnetismo pelo seu potencial no desenvolvimento de aplicações em engenharia óptica, de microondas e milimétrica.

As influências de um meio bi-isotrópico na propagação de ondas eletromagnéticas pode ser decomposta em efeito de quiralidade e efeito Tellegen. Meios puramente quirais, também denominados meios Pasteur ou meios quirais recíprocos, são birrefringentes, ou melhor. apresentam duas "autofunções de onda" circulamente polarizadas em sentidos opostos que enxergam o meio bi-isotrópico como meios isotrópicos distintos. A inclusão do efeito Tellegen para expressar meios bi-isotrópicos é um assunto polêmico. Enquanto meios quirais são encontrados em abundância na natureza e podem ser fabricados artificialmente, ainda não há a comprovação da existência de meios Tellegen biisotrópicos. A meios caracterizados pelo efeito Tellegen seriam adicionadas as propriedades de bi-impedância e nãoreciprocidade [1].

Os autores são da Universidade Federal do Pará (UFPA), DEE, CEP. 66075-900 Belém - PA. Este trabalho foi parcialmente financiado pela CAPES e pelo CNPq.
O método das diferenças finitas no domínio do tempo vem se tornando uma das técnicas numéricas eletromagnéticas mais usadas, devido à facilidade de compreensão e implementação e. por ser uma técnica no domínio do tempo, é capaz de abranger uma extensa faixa de freqüências numa única execução pela utilização de um pulso de excitação no domínio do tempo (como um pulso gaussiano). Isto é extremamente útil em aplicações onde as freqüências de ressonância não são exatamente conhecidas. ou toda vez que um resultado em banda larga é desejado.

A finalidade deste trabalho é propor um modelo baseado no método FDTD e testar sua aplicabilidade na simulação e análise da propagação de ondas eletromagnéticas em guias de ondas contendo materiais bi-isotrópicos, principalmente quirais. Consequentemente, visa-se também a obtenção de uma ferramenta numérica simples e versátil para auxiliar o projeto de dispositivos de telecomunicações contendo estes materiais e designados para operar na banda milimétrica, onde é esperado que o progresso na ciência de polímeros proporcionará o uso comum de materiais quirais em tecnologia futura.

\section{METODOLOGIA NUMÉRICA}

Dentre as variaçōes existentes para a representação das relações constitutivas para meios bi-isotrópicos. as expressões mais satisfatórias no que tange à estabilidade do método das Diferenças Finitas no Domínio do Tempo (FDTD) são dadas por

$$
\begin{aligned}
& \vec{D}=\varepsilon \vec{E}+\frac{1}{c_{0}}(\chi-j \kappa) \vec{H}, \\
& \vec{B}=\mu \vec{H}+\frac{1}{c_{0}}(\chi+j \kappa) \vec{E},
\end{aligned}
$$

onde $\varepsilon$ é a permissividade elétrica do meio, $\mu$ é permeabilidade magnética, $c_{0}$ é a velocidade da luz no vácuo, $\kappa$ é o parâmetro de quiralidade (adimensional) e $\chi$ é o parâmetro Tellegen (adimensional). Materiais com $\chi \neq 0$ não são recíprocos [1]. Apesar de que as relações constitutivas estejam caracterizadas no domínio da freqüência (convenção temporal $\mathrm{e}^{\mathrm{j \omega t}}$ ), estas podem ser aplicadas ao método FDTD considerando que a variação dos parâmetros dos materiais $(\varepsilon, \mu, \kappa, \chi)$ seja desprezível na faixa de freqüência analisada.

Aqui serão consideradas as soluções recursivas para os meios bi-isotrópicos. As equações de Maxwell necessárias são:

$$
\frac{\partial \vec{B}}{\partial t}=-\nabla \times \vec{E}
$$




$$
\frac{\partial \vec{D}}{\partial t}+\sigma \vec{E}=\nabla \times \vec{H} .
$$

Visando a minimização do acúmulo de erros de processamento, as seguintes normalizaçōes são empregadas em (1)-(4):

$$
\bar{E}=\vec{E}, \quad \bar{H}=\eta_{0} \vec{H}, \quad \bar{D}=\vec{D} / \varepsilon_{0}, \quad \bar{B}=c_{0} \vec{B}
$$

onde $\eta_{0}$ é a impedância intrínseca do vácuo, $\varepsilon_{0}$ é a permissividade elétrica absoluta do vácuo e $c_{0}$ é a velocidade da luz no vácuo. Considerando as equações (1)(4) normalizadas e eliminando os vetores $\bar{D}$ e $\bar{B}$, obtém-se as seguintes equações:

$$
\begin{gathered}
\mu_{r} \frac{\partial \bar{H}}{\partial t}+\zeta_{r} \frac{\partial \bar{E}}{\partial t}=-c_{0} \nabla \times \bar{E} \\
\varepsilon_{r} \frac{\partial \bar{E}}{\partial t}+\xi_{r} \frac{\partial \bar{H}}{\partial t}=c_{0} \nabla \times \bar{H} .
\end{gathered}
$$

onde $\xi_{r}=\zeta_{r}^{*}=(\chi-j \kappa)$. Isolando as derivadas temporais dos campos elétrico e magnético em equações distintas e aplicando o método FDTD na discretização temporal destes campos, obtém-se as suas soluções recursivas, atualizadas a cada passo temporal $\delta t$ :

$$
\begin{aligned}
\bar{H}^{n+\frac{1}{2}}=\bar{H}^{n-\frac{1}{2}}-c_{0} \delta t\left(\frac{\varepsilon_{r}}{\mu_{r} \varepsilon_{r}-\xi_{r} \zeta_{r}}\right)\left(\nabla \times \bar{E}^{n}+\frac{\zeta_{r}}{\varepsilon_{r}} \nabla \times \bar{H}^{n}\right) \\
\bar{E}^{n+1}=\bar{E}^{n}+c_{0} \delta t\left(\frac{\mu_{r}}{\mu_{r} \varepsilon_{r}-\xi_{r} \zeta_{r}}\right)\left(\nabla \times \bar{H}^{n+\frac{1}{2}}+\frac{\xi_{r}}{\mu_{r}} \nabla \times \bar{E}^{n+\frac{1}{2}}\right)
\end{aligned}
$$

Decorre porém. que o método FDTD não prevê amostras do campo magnético no instante $n \delta t$ e nem amostras do campo elétrico no instante $(n+1 / 2) \delta t$, necessários para computar o efeito de quiralidade a partir dos rotacionais do campo magnético em (8) e do rotacional do campo elétrico em (9). São feitas, dessa forma, as seguintes aproximaçōes, considerando $\delta t$ suficientemente pequeno (com valor menor que o estimado através do critério de estabilidade para estruturas simples):

$$
\nabla \times \bar{H}^{n} \approx \nabla \times \bar{H}^{n-\frac{1}{2}}, \quad \nabla \times \bar{E}^{n+\frac{1}{2}} \approx \nabla \times \bar{E}^{n}
$$

No processo discretização espacial, são realizadas aproximações sobre as derivadas espaciais presentes nos rotacionais dos campos. Tais derivadas. dependendo da geometria do problema, podem apresentar dedução analítica ao longo de um determinado eixo, eliminando a necessidade de discretizar a estrutura segundo este eixo, o que se torna vantajoso em termos de tempo de processamento. Por exemplo, para estruturas com seção transversal invariável segundo o eixo $z$, os rotacionais discretizados espacialmente, tendo como referência a célula de Yee bidimensional mostrada na Fig.1, são dados por (2DFDTD):

$$
\nabla \times \bar{E} \approx\left(\begin{array}{c}
\frac{1}{\delta y}\left[E_{z(I . J+1)}-E_{z(I . J)}\right]+j \beta E_{y\left(I . J+\frac{1}{2}\right)} \\
-j \beta E_{y\left(I+\frac{1}{2} J\right)}-\frac{1}{\delta x}\left[E_{z(I+1 . J)}-E_{z(I . J)}\right] \\
\frac{1}{\delta x}\left[E_{y\left(I+1 . J+\frac{1}{2}\right)}-E_{y\left(I J J+\frac{1}{2}\right)}\right]-\frac{1}{\delta y}\left[E_{x\left(I+\frac{1}{2} J+1\right)}-E_{x\left(I+\frac{1}{2} J\right)}\right]
\end{array}\right)
$$

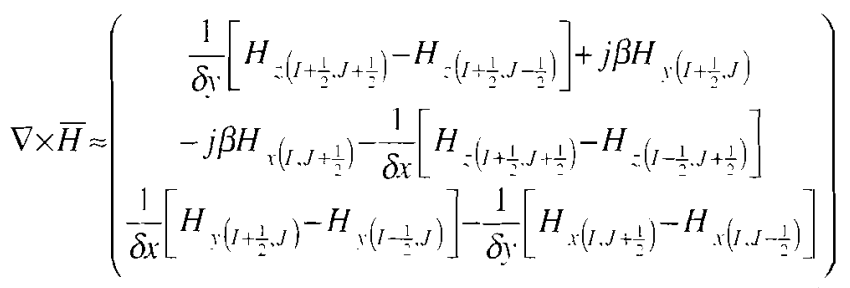

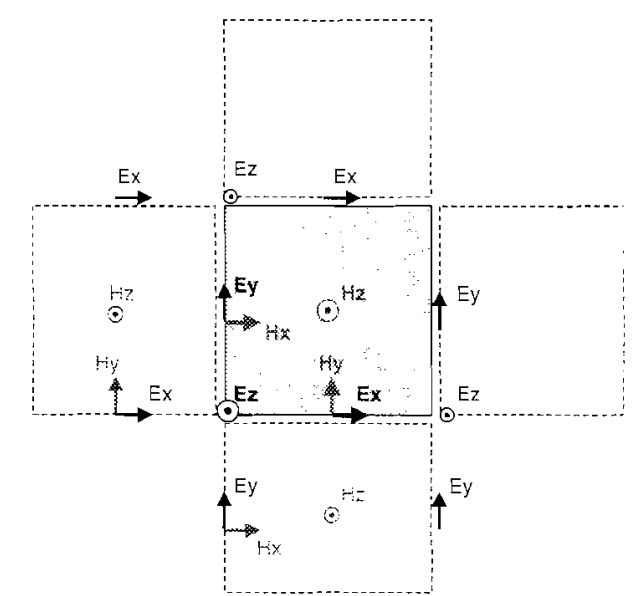

Figura 1. Célula para discretização bidimensional.

\section{RESULTADOS}

Com base nas soluções numéricas obtidas. um fonte é implementado para analisar as característices iz propagação de algumas estruturas guiadas que apresentam material quiral, para demonstrar a validade e a abrangência do modelo FDTD desenvolvido.

\subsection{GUIA QUIRAL DE PLACAS PARALELAS METÁLICAS}

Para a testar validade da formulação apresentada. o modelo de estrutura utilizado inicialmente nas simulações é o guia de ondas quiral de placas paralelas, mostrado na Fig. 2. Na discretização espacial foi utilizado o esquema unidimensional, que é uma extensão da idéia utilizada na discretização bidimensional à estruturas que também apresentam uniformidade em relação a um segundo eixo cartesiano. A fim de implementar as camadas metálicas foram usadas células condutoras através do tratamento numérico das soluções homogênea e particular para o campo elétrico em meios com condutividade elevada. conforme exposto em [2]. A Fig. 3 mostra detalhes da discretização.

Com relação a outros valores de parâmetros empregados, a altura do núcleo é $h=1.0 \mathrm{~mm}$, a dimensão mínima da célula de discretização espacial é $\delta \mathrm{min}=0,05 \mathrm{~mm}$. a altura do domínio numérico é $L_{Y}=1,4 \mathrm{~mm}$, o número de células 
ao longo do eixo y é $n y=28$ células. Estes são os parâmetros da estrutura e discretização espacial.

Referindo-se aos parâmetros do pulso de excitação gaussiano, o seu valor rms segundo o eixo y é $\sigma_{r}=0,5 \mathrm{~mm}$ e a largura de banda do pulso de excitação é $B_{f}=562 \mathrm{GHz}$.

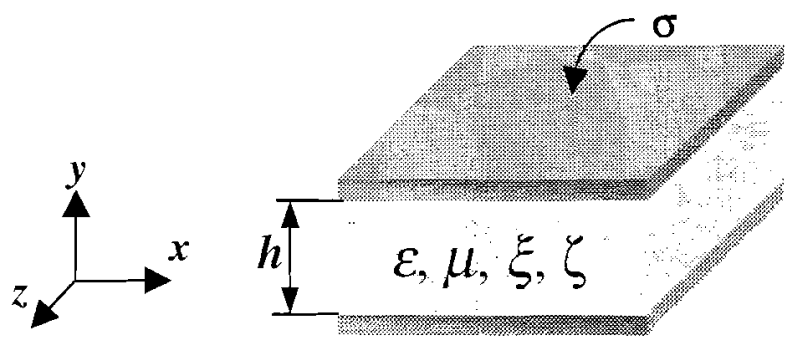

Figura 2. Guia quiral de placas paralelas.

Sobre os parâmetros da discretização temporal, o número de passos temporais para cada ponto na curva de dispersão é $n t=6001$ e a constante de ajuste do incremento temporal é $C_{t}=0,1$. Este último valor significa que, para garantir a estabilidade do método FDTD, foi utilizado um passo temporal $(\delta t)$ dez vezes menor em relação ao que seria utilizado num meio convencional pela condição de estabilidade de Courant.

Os valores da constante de fase $\beta$ ( $\mathrm{rad} . / \mathrm{mm})$ para a formação da curva de dispersão $(\beta z=\beta, \beta x=0)$ são $\beta=$ 0,$5 ; 1 ; 2 ; 3 ; 4 ; \ldots 10$.

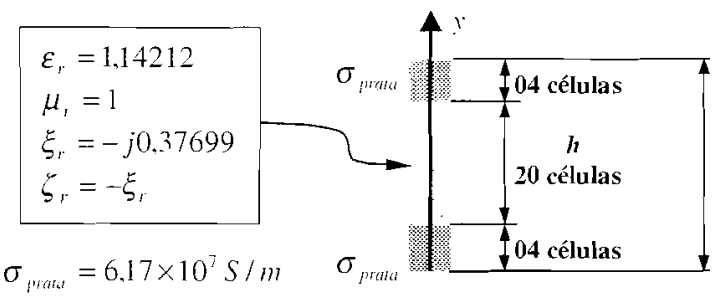

Figura 3. Discretização espacial unidimensional do guia.

As características obtidas são ilustradas na Fig. 4 conjuntamente aos resultados fornecidos em [3], onde $\Omega=\omega h \sqrt{\mu \varepsilon}$. Observa-se que os resultados são concordantes próximo às freqüências de corte do guia, onde são constatadas uma bifurcação e o modo dominante, de freqüência de corte nula. Porém, para valores mais elevados de constante de fase normalizada $\beta h$, as curvas tendem a divergir em relação à literatura. Isto pode estar relacionado ao fato de que. no meio quiral, por haver o acoplamento cruzado entre o campo elétrico e o campo magnético, assim como a aproximação dos modos devido a ocorrência de bifurcações, poderá ocorrer a autoexcitação de outros modos, principalmente se modos vizinhos apresentarem semelhanças nas configurações espaciais de campo. Isto poderá resultar na dificuldade ou impossibilidade de obtenção da freqüência modal a partir do registro temporal e gerar algum erro nas curvas de dispersão.

Segundo análise realizada em [4] acerca das distribuições espaciais para modos bifurcados, ramos inferiores de bifurcações correspondem a modos ímpares, enquanto que ramos superiores correspondem a modos pares. Conciliando este fundamento à aplicação do método FDTD, foram utilizadas excitações teoricamente favoráveis a extração e análise de cada modo híbrido.

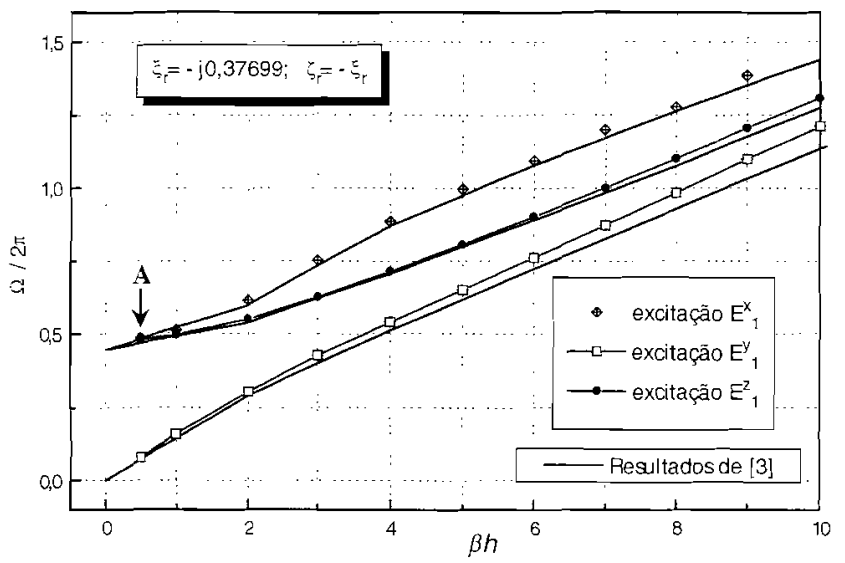

Figura 4. Diagrama de Brillouin para modos propagantes no guia quiral de placas paralelas.
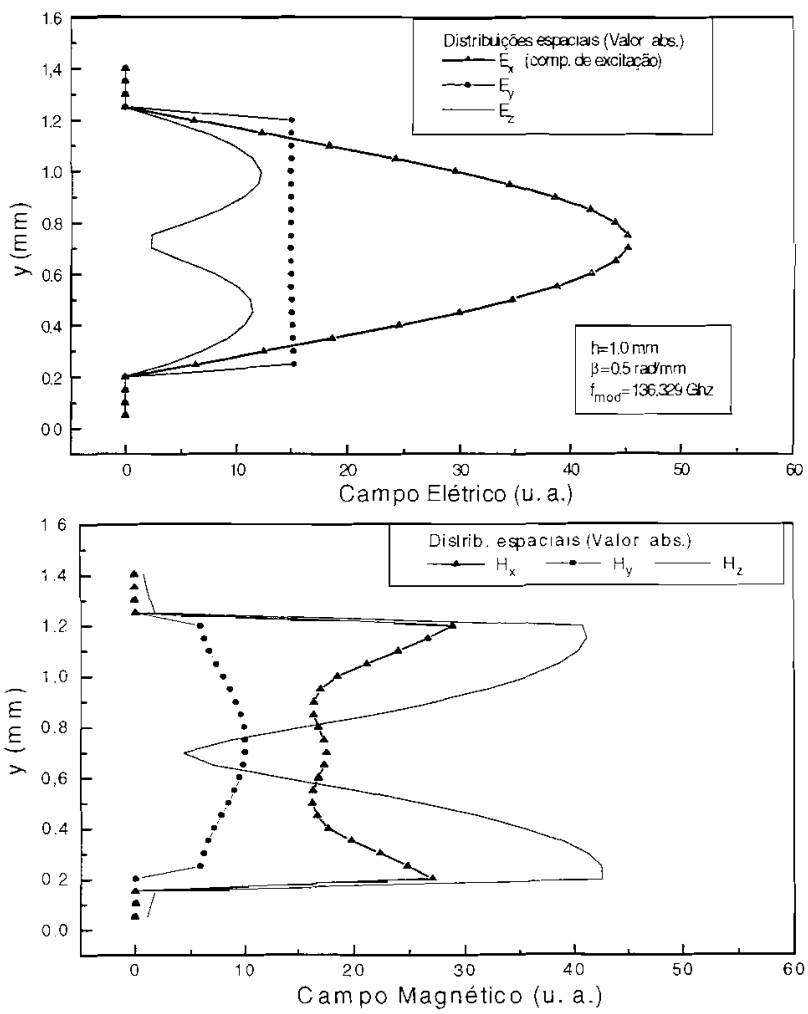

Figura 5. Distribuição espacial das componentes de campo elétrico e magnético relativas à excitação da componente Ex para um baixo valor de constante de fase $\beta$ nos casos de guia de placas paralelas infinitas quiral $\left(\xi_{r}=-j 0,37699 ; \zeta_{r}=-\xi_{r}\right)$.

A Fig. 5 mostra a distribuição espacial do valor médio absoluto das componentes de campo elétrico e magnético correspondente a um baixo valor de constante de fase $\beta$ (relativo ao ponto A da Fig. 4), próximo a região de corte do modo, para evitar a interferência de outros modos que possam ser auto excitados devido a complexidade do meio. A componente de campo elétrico $E_{x}$ é excitada temporariamente como um pulso gaussiano no tempo e segundo a direção y. A Fig. 6 corresponde às condições da 

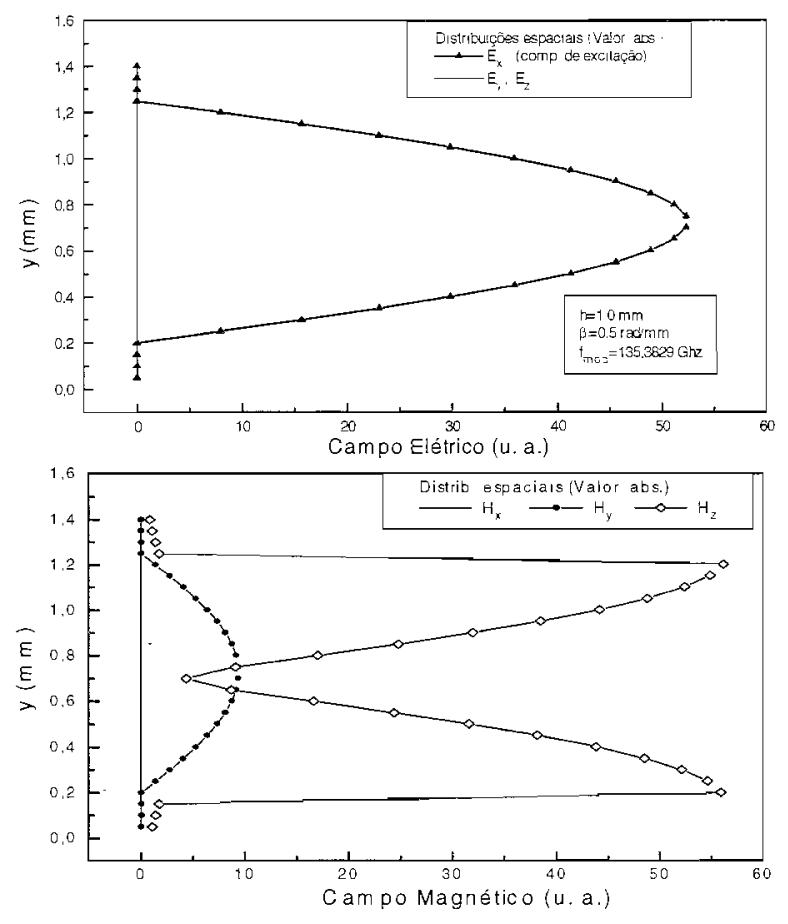

Figura 6. Distribuição espacial das componentes de campo elétrico e magnético relativas à excitação da componente Ex para um baixo valor de constante de fase $\beta$ no caso de guia de placas paralelas infinitas aquiral (modo TE).

Fig. 5, exceto que na primeira os parâmetros de quiralidade são igualados a zero.

A Fig. 7 mostra a distribuição espacial do valor médio absoluto das componentes de campo elétrico e magnético correspondentes a um baixo valor de constante de fase $\beta$ (relativo também ao ponto A da Fig. 4). Neste caso. a componente de campo elétrico excitada é $E_{-}$. A Fig. 8 corresponde às condições da Fig. 7, exceto que na primeira os parâmetros de quiralidade são igualados a zero.

Apesar de que as distribuições espaciais mostradas na Fig. 5 e Fig. 7 correspondam a modos que possuem a mesma freqüência de corte (coincidindo, portanto, no ponto A da Fig. 4), o primeiro conjunto de distribuições, onde a excitação inicial aplicada é $E_{j}^{x}$, correspondente ao ramo superior da bifurcação da Fig. 4 (modo par), enquanto que o segundo conjunto, onde a excitação inicial empregada é $E_{i}^{i}$, corresponde ao seu ramo inferior (modo ímpar). Além do mais, quando a quiralidade do meio tende a zero, um dos modos da bifurcação tende a assumir a configuração do TE convencional, enquanto que o outro tende a adquirir o aspecto do modo TM convencional, conforme pode ser observado na Fig. 6 e Fig. 8, respectivamente. Consequentemente, estes resultados estão de acordo com o estudo desenvolvido em [4]. O modo dominante do guia quiral de placas paralelas, de freqüência de corte nula, considerado inexistente em [4], é extraído e analisado em trabalhos comparativamente mais recentes, como em [3] e [5]. Este modo tende ao convencional TEM quando a quiralidade do meio tende a zero.

Pela utilização de células metálicas para implementar as placas, as condições de contorno na interface entre o metal e
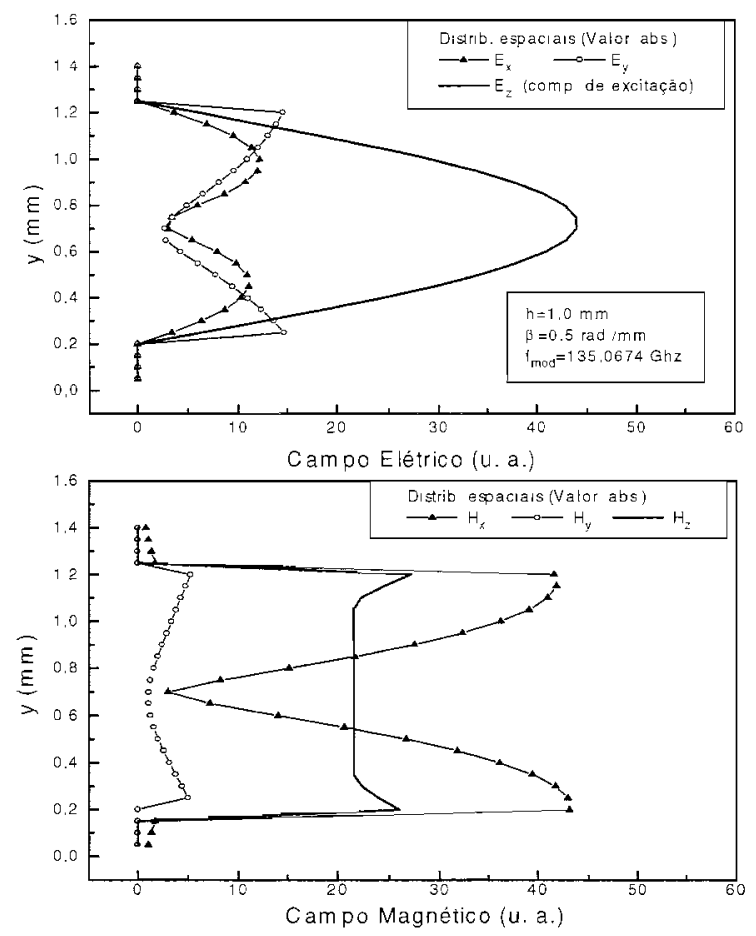

Figura 7. Distribuição espacial das enmnnnentz: t구 campo elétrico e magnético relativas à excitação da componente $E_{z}$ para um baixo valor de constante de fase $\beta$ no caso de guia quiral de placas paralelas infinitas
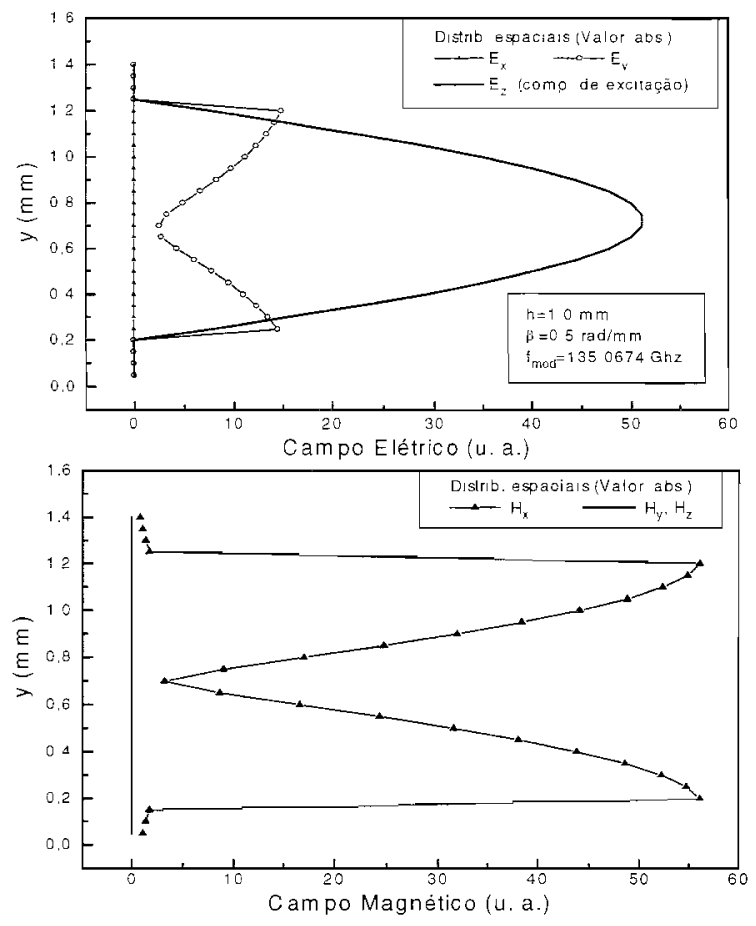

Figura 8. Distribuição espacial das componentes de campo elétrico e magnético relativas à excitação da componente $E z$ para um baixo valor de constante de fase $\beta$ no caso de guia de placas paralelas infinitas aquiral (modo TM). 
o interior do guia são atingidas de uma forma natural no processo iterativo, pelas diferenças nas soluções recursivas dos campos em cada região. Observe pela Fig. 5 que a componente de campo magnético $H_{y}$ na interface entre metal e meio quiral não é nula como na interface entre metal e dielétrico comum (aquiral).

A respeito da velocidade de processamento, considerando a discretização espacial empregada (número de células), o número de passos temporais e a máquina utilizada, sendo esta última uma estação IBM com processador Pentium II de $266 \mathrm{MHz}$ de clock, o tempo de execução para cada ponto tomado individualmente no diagrama da Fig. 4 é de 38,5 segundos.

\subsection{GUIA QUIRAL CIRCULAR DIELÉTRICO}

Desta vez é utilizado o modelo de discretização bidimensional (2D-FDTD) para computar as características de propagação de um guia quiral circular dielétrico imerso no ar. Em se tratando de material bi-isotrópico, onde ondas circularmente polarizadas são auto-soluções em espaço aberto. esta geometria aparenta ser a mais promissora para a prática de engenharia [1]. O guia de ondas circular e sua discretização espacial são mostrados na Fig. 9.

Os parâmetros do material do guia são $\varepsilon_{1}=1,2419 \varepsilon_{0}$. $\mu_{1}=\mu_{0}$ e $\xi_{\mathrm{r}}=-\mathrm{j} 0,37673$, os quais são equivalentes aos empregados em [6], porém com valores adaptados às relações constitutivas usadas neste trabalho, a fim de possibilitar a comparação de resultados.

Como se pode observar pela Fig. 9(b). é atribuído um raio $\mathrm{R}=1 \mathrm{~mm}$ ao guia, onde também é reservada uma folga mínima de $1,5 \mathrm{~mm}$ entre sua borda circular'e os limites do domínio espacial numérico, que é contornado pela parede absorvente de Mur ( $1^{\text {a }}$ ordem). para eliminar erros provocados por reflexôes nos limites.

Para construir o perfil circular na malha bidimensional de células retangulares, faz-se uso da seguinte inequação, a qual deve ser satisfeita para o preenchimento da célula com o material do guia:

$$
\sqrt{D_{x_{1}}^{2}+D_{y_{1}}^{2}} \leq R
$$

onde

$$
\begin{aligned}
& D_{x_{I}}=\left(I-I_{r p}\right) \delta_{\min }-\delta_{m i n} / 2, \\
& D_{y_{J}}=\left(J-J_{\eta p}\right) \delta_{m i n}-\delta_{m i n} / 2 .
\end{aligned}
$$

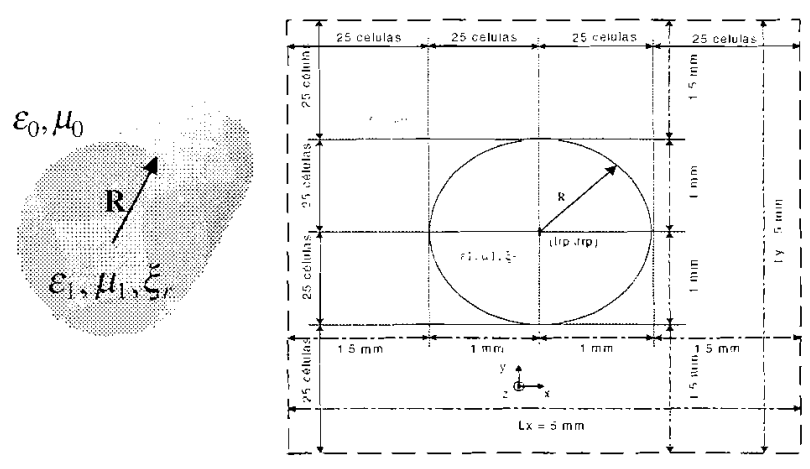

Figura 9. Ilustrações do (a) Guia circular quiral e (b) discretização espacial.
As distâncias horizontal e vertical são medidas a partir do ponto de referência ( $I r p, J r p$ ) no centro do guia até uma coordenada genérica de célula $(I, J)$ no domínio espacial. O parâmetro $\delta m i n$ é a dimensão da largura e da altura da célula na região do guia. Nesta região deve ser usada uma definição satisfatória (número de células grande o suficiente) para que o perfil circular seja caracterizado.

A seguir são listados valores não referenciados de parâmetros usados na simulação, considerando a presente estrutura.

A respeito dos parâmetros da estrutura e discretização espacial, $\delta$ min $=0,04 \mathrm{~m}$, a largura e altura do domínio numérico é $L_{X}=L_{Y}=5 \mathrm{~mm}$; o número de células ao longo dos eixos $x$ e yé $n x=n y=100$.

$O$ valor rms do pulso de excitação gaussiano segundo o eixo y é $\sigma_{y}=1 \mathrm{~mm}$, enquanto que, segundo o eixo $\mathrm{x}$ foi utilizada uma função gaussiana de valor $r m s \sigma_{x}=1 \mathrm{~mm}$ na excitação do modo $H E_{1,1}$ e duas funções gaussianas com $\sigma_{x}=0,2 \mathrm{~mm}$ na excitação do modo $H E_{2.1}$. A largura de banda do pulso de excitação é $B_{f}=421 \mathrm{GHz}$.

Sobre os parâmetros da discretização temporal, o número de passos temporais é nt $=4001$ e a constante de ajuste do incremento temporal é $C_{t}=0,25$.

Os valores da constante de fase $\beta$ ( $\mathrm{rad} . / \mathrm{mm}$ ) usados para a formação da curva de dispersão são $\beta=1,3$ (apenas $\left.H E_{1,1}\right): 2: 3: 4: 5 \ldots 12$.

A Fig. 10 mostra as características de propagação do modo dominante $H E_{1.1}$ e do modo $H E_{2,1}$. A notação $H E_{m, m}$ é usada para designar um modo híbrido onde $n$ e $m$ são números modais azimutal e radial. respectivamente [7]. Os pontos referem-se aos resultados obtidos através do modelo. FDTD apresentado neste trabalho, enquanto que as curvas contínuas são os resultados exatos descritos em [6].

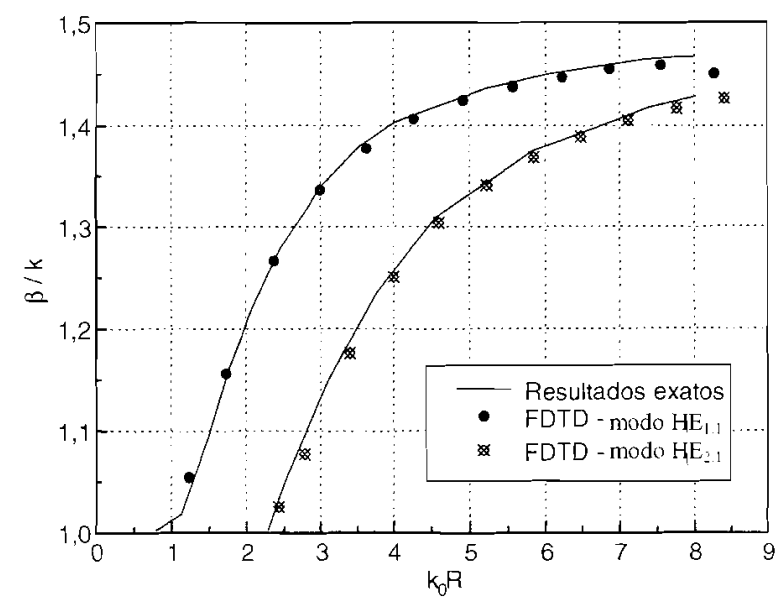

Figura 10. Características de dispersão do guia quiral circular dielétrico.

As características espaciais dos pulsos de excitação utilizados na obtenção de cada modo são mostrados na Fig. 11. Esses pulsos são gaussianos, onde $\sigma_{x}$ e $\sigma_{y}$ são os valores rms segundos os eixos $x$ e $y$. respectivamente. No tempo são usadas duas funções gaussianas com atrasos distintos e sinais opostos.

A Fig. 12 mostra a resposta espectral da componente $E_{\mathrm{y}}$ para $\beta=3 \mathrm{rad} / \mathrm{mm}$ e excitação do $\operatorname{modo} H E_{1.1}$, 
correspondente ao ponto $(x=2,36953 ; y=1,26608)$ no gráfico de dispersão da Fig. 10, onde constata-se a presença de uma única solução na freqüência de $113.0583 \mathrm{GHz}$. A fim de obter a natureza da polarização deste modo, o ângulo entre campo elétrico e o ejxo $x$ pode ser obtido pela expressão $\theta_{x}=(180 / \pi) \arctan \left(E_{y} / E_{x}\right)$. o que é realizado no centro do guia e ilustrado na Fig. 13. para cada instante de tempo. Lembrando que a excitação apresenta polarização linear segundo o eixo y, num meio aquiral este ângulo alternaria entre $90^{\circ}$ e $-90^{\circ}$ a cada meio período. Através da Fig. 13 pode-se concluir que no material quiral, após a remoção do pulso de excitação a polarização do campo elétrico estabiliza girando continuamente no sentido horário, olhando no sentido do eixo de propagação. A ocorrência de um único modo na resposta espectral e a rotação uniforme do campo estabilizado no sentido horário evidenciam a presença de apenas uma das duas soluções naturais do meio bi-isotrópico, a que apresenta polarização circular no sentido horário, distinguida pelo subscrito "+" [1],[8]. O valor do índice de refração equivalente para esta solução, obtido através da análise de ondas planas propagando em meios bi-isotrópicos, é de $n_{+}=\sqrt{\mu_{r} \varepsilon_{r}}+\kappa=$ $\sqrt{1,24919}+0.37673 \approx 1,4911$, que é o valor assintótico das curvas de dispersão no gráfico da Fig. 10. O índice de refração equivalente no meio quiral ilimitado para a onda circularmente polarizada no sentido anti-horário é $n_{-}=\sqrt{\mu_{r} \varepsilon_{r}}-\kappa \approx 0,7409$, sendo menor que o índice de refração no meio externo (ar), o que explicaria a sua não ocorrência neste dispositivo.

O tempo de processamento de cada ponto na curva de dispersão da Fig. 10 na estação IBM com processador Pentium II de $266 \mathrm{MHz}$ de clock é de 33,35 minutos, que é consideravelmente maior que o tempo referente ao guia de ondas de placas paralelas (38,5 segundos), devido à passagem do esquema unidimensional ao bidimensional, havendo um grande aumento no número de células para a discretização da estrutura. ou seja, para o guia de placas paralelas são utilizadas $n_{y}=28$ células enquanto que para o guia circular o número de células é $n_{x} \times n_{y}=100 \times 100=$ 10000

\subsection{GUIA QUIRAL CIRCULAR DIELÉTRICO MULTICAMADAS}

O mérito do método FDTD, além de sua simplicidade em relação a outros métodos numéricos, é a flexibilidade no que diz respeito às geometrias de estruturas aptas a serem discretizadas para simulação, facilitando a análise de estruturas com perfis complexos onde o tratamento é complicado ou até mesmo impossível através de outras técnicas. Um exemplo de tal estrutura é o guia quiral circular multicamadas, proposto neste trabalho e mostrado na Fig. 14. A estrutura simulada é composta por três regiões circulares concêntricas, referidas do centro para a extremidade como núcleo, tubo e casca. onde o efeito de quiralidade é analisado para cada região.

Os valores de parâmetros usados são $\varepsilon_{1}=4 \varepsilon_{0}, \varepsilon_{2}=$ $\varepsilon_{3}=12,6 \varepsilon_{0}, \mathrm{Ra}=1 \mathrm{~mm}, \mathrm{Rb}=0,75 \mathrm{~mm}$ e $\mathrm{Rc}=0,5 \mathrm{~mm}$. A permeabilidade magnética é $\mu=\mu 0$ em todo o espaço.

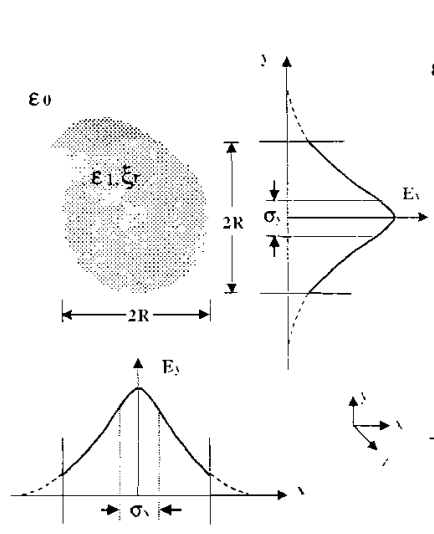

(a)

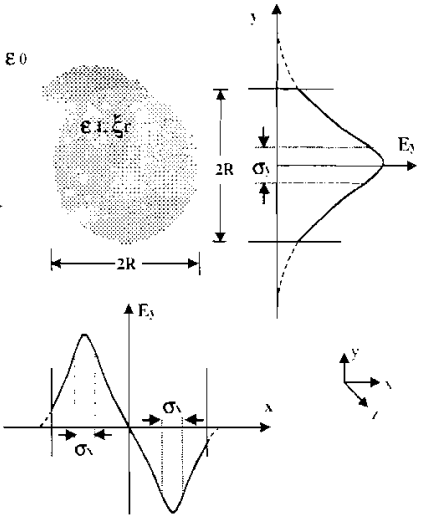

(b)
Figura 11. Distribuições espaciais dos pulsos gaussianos usados na excitação dos modos (a) $H E_{1.1}$ e (b) $H E_{2.1}$.

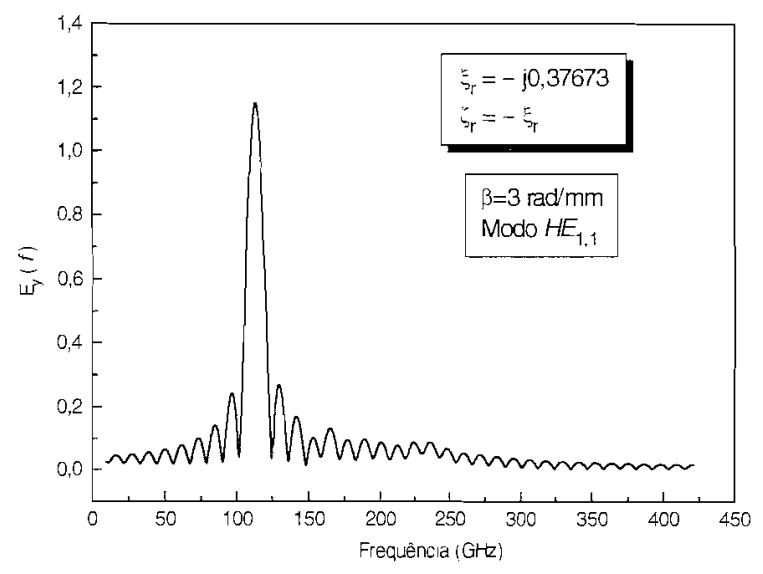

Figura 12. Resposta espectral para $\beta=3 \mathrm{rad} / \mathrm{mm} \mathrm{e}$ excitação do modo $H E_{1.1}$.

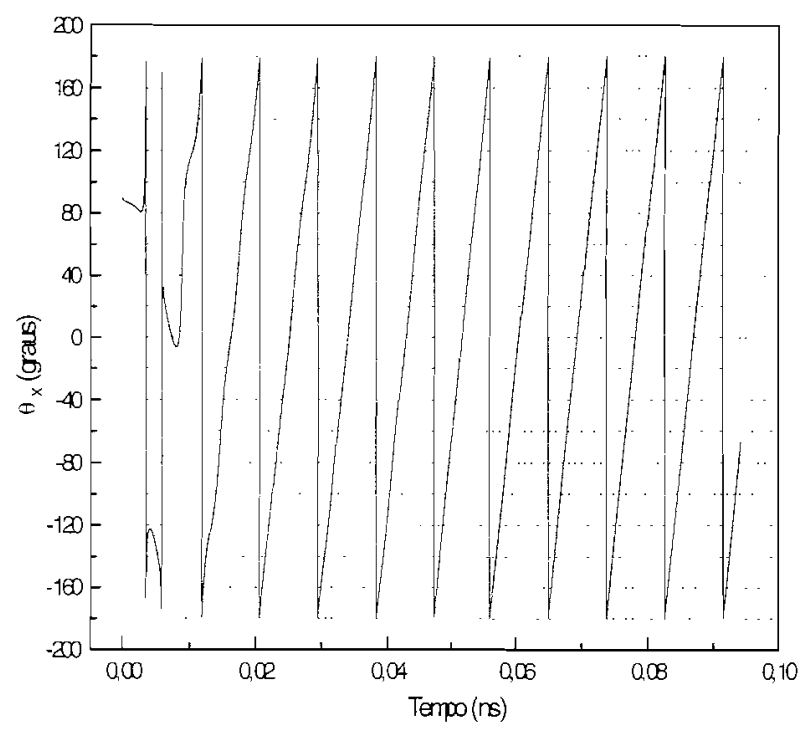

Figura 13. Comportamento temporal da orientação do campo elétrico no plano xy $(\beta=3 \mathrm{rad} / \mathrm{mm}$ e excitação do modo HE1,1). O ângulo é medido da forma usual, a partir do eixo $x$. 


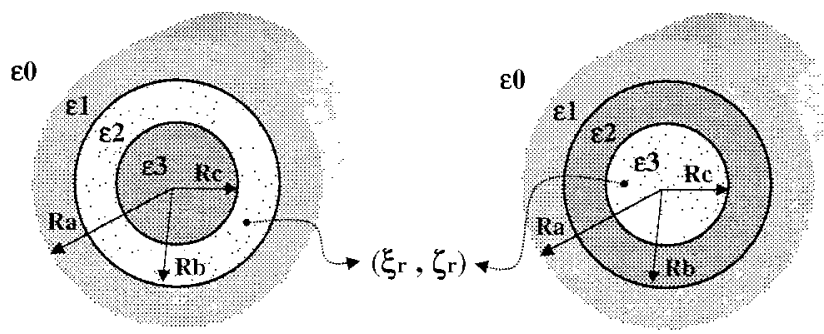

(b)

Figura 14. Guia circular dielétrico multicamadas contendo material quiral (a) no tubo e (b) no núcleo.

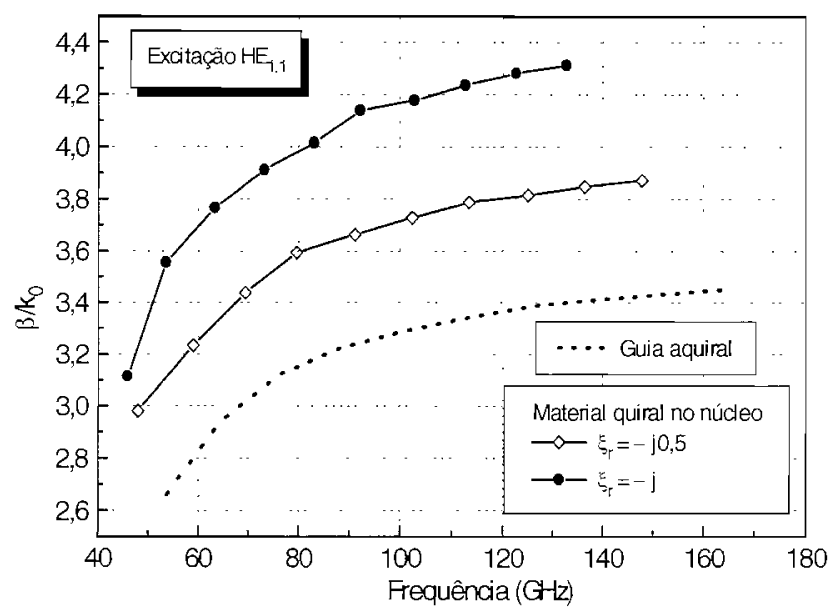

Figura 15. Características de propagação para o guia circular com material quiral no núcleo.

As simulações foram realizadas para dois valores de $\xi_{1}$ : $0,5 \mathrm{j} \mathrm{e}-\mathrm{j}$.

A excitação é o modo dominante $\mathrm{HE}_{1 . \mathrm{I}}$. Os valores da constante de fase de entrada para construir as curvas de dispersão são $\beta=3 ; 4 ; 5 ; 6 ; \ldots 12 \mathrm{rad} / \mathrm{mm}$. O número de iterações no tempo é nt $=5001$. Aos demais parâmetros, relacionados à discretização, são atribuídos valores similares aos utilizados para o guia circular.

A curva de dispersão no caso em que o guia circular apresenta material quiral no núcleo é ilustrada na Fig. 15, onde a curva da versão aquiral do guia é exposta para fins comparativos. As características de propagação associadas ao guia com material quiral na casca não estão incluídas por não apresentarem nenhuma alteração visível em relação ao caso aquiral, considerando que os campos são fracos nesta região devido ao alto contraste entre o índice de refração da casca e o índice das regiões internas.

Para o caso no qual o núcleo contém material quiral, o aumento do índice de refração efetivo com o aumento do parâmetro de quiralidade é observado (Fig. 15), indicando que o modo circularmente polarizado no sentido horário se impôs pela magnitude.

A Fig. 16 ilustra as respostas espectrais relativas ao guia aquiral e ao guia com material quiral no núcleo para $\beta=4$ $\mathrm{rad} . / \mathrm{mm}$, onde observa-se que o modo dominante para o

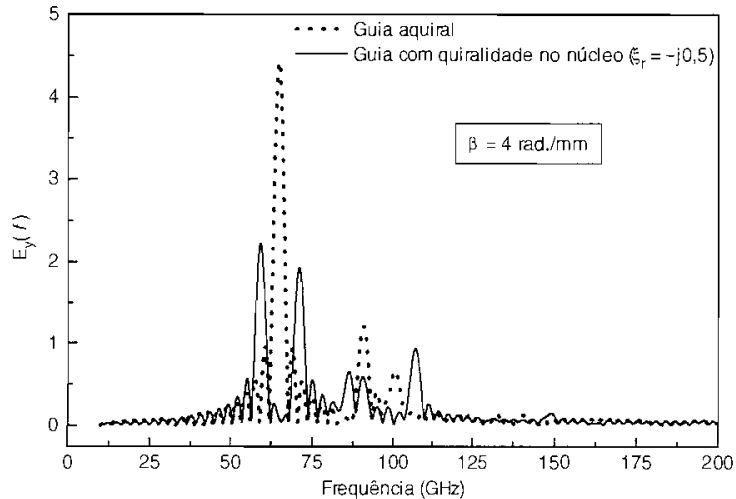

Figura 16. Respostas espectrais do guia em análise nos casos aquiral e com quiralidade no núcleo, para $\beta=4 \mathrm{rad} . / \mathrm{mm}$.

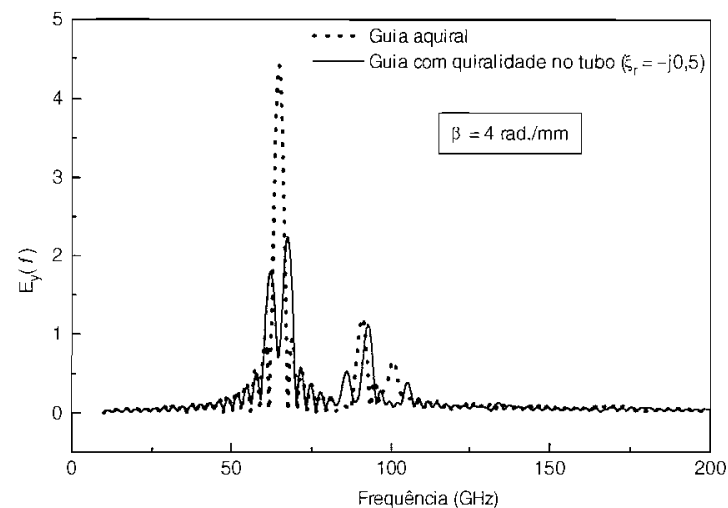

Figura 17. Respostas espectrais do guia em análise, nos casos aquiral e com quiralidade no tubo, para $\beta=4 \mathrm{rad} . \mathrm{mm}$.

caso aquiral (linearmente polarizado) é decomposto em dois modos próximos, onde o modo com freqüência menor seria a solução circularmente polarizada no sentido horário, que se impôs pela intensidade, enquanto que o modo com freqüência maior seria a solução circularmente polarizada no sentido anti-horário.

A Fig. 17 compara a resposta espectral do guia circular multicamadas de tubo quiral com a resposta espectral do guia aquiral para $\beta=4 \mathrm{rad} . / \mathrm{mm}$. onde a decomposição do modo dominante em dois pela inserção da quiralidade também é verificada, sendo que desta vez o modo circularmente polarizado no sentido anti-horário se impôs pela intensidade. Isto provavelmente se deve ao fato de que ondas circularmente polarizadas no sentido horário passam a enxergar o tubo com um índice de refração maior que o do núcleo, distribuindo mais a sua energia na região do tubo em relação à ondas circularmente polarizadas no sentido anti-horário, que enxergam o tubo com um índice de refração equivalente menor e se confinam mais no núcleo, onde são realizadas as medidas do campo elétrico.

O tempo de processamento para a obtenção de cada ponto nas curvas de dispersão da Fig. 15, em uma estação IBM com processador Pentium II de $266 \mathrm{MHz}$ de clock, é de 38.84 minutos 


\section{CONCLUSÃO}

Neste trabalho, estratégias de simulação baseadas no método numérico FDTD são propostas e utilizadas na análise de problemas eletromagnéticos envolvendo estruturas propagantes contendo materiais bi-isotrópicos, especialmente quirais, os quais vêm, recentemente. despertando grande interesse na área de eletromagnetismo aplicado. devido às suas aplicações em potencial na área de dispositivos ópticos integrados, sistemas de comunicações, etc. O método FDTD é conhecido pela sua simplicidade e flexibilidade no modelamento devido à possibilidade de ser empregada diretamente às equações de Maxwell

A questão básica a respeito da aplicabilidade do método FDTD a meios bi-isotrópicos é averiguada através da simulação de estruturas cujas características de propagação estão disponibilizadas na literatura através de outras técnicas, como o guia quiral de placas paralelas e o guia quiral dielétrico circular, onde foi constatado que os resultados obtidos através do método FDTD são satisfatoriamente compatíveis, dentro da faixa de freqüência analisada. Da mesma forma, resultados para o guia quiral circular multicamadas são incluídos para ilustrar que o método FDTD pode ser usado na simulação de dispositivos com geometria mais elaborada, alterando apenas a discretização espacial no código fonte existente. Esta é a principal contribuição que um modelo baseado no método FDTD pode proporcionar, principalmente quando são envolvidos materiais complexos, cujo modelamento pode ser complicado ou até mesmo impossível através de outros meios.

Prosseguindo a discussão sobre a aplicabilidade do método, vale lembrar que o esquema de discretização usual do método FDTD, de campos intercalados no tempo e no espaço, não prevê o caso em que bi-isotropia é inserida, o que impõe a necessidade de realizar discretizações levemente desviadas do ponto ideal, em cima das componentes de campo que acoplam devido aos parâmetros de bi-isotropia, para proporcionar o enquadramento destas componentes nesse esquema de discretização. Por sua vez, isto requer a adoção de um critério de estabilidade mais rigoroso que o convencional para a escolha de um passo temporal $(\delta t)$ adequado a este modelo. A necessidade de utilizar um passo temporal menor em comparação a meios isotrópicos simples eleva o processamento relativo a uma determinada estrutura, pois para abranger um determinado tempo em um problema de eletromagnetismo, é requisitado um número maior de passos temporais.

\section{REFERÊNCIAS}

[1] I. Lindell, A. Sihvola. S. Tretyakov. and A. Viitanen. Electromagnetic Waves in Chiral and Bi-Isotropic Media. Artech House. 1994.

[2] A. Taflove. Computational Electrodynamics - The FiniteDifference Time-Domain Method. Artech House, 1995.

[3] G. Plaza. F. Mesa. and M. Horno, "Computation of propagation characteristics of chiral layered waveguides". IEEE Trans. Microware Theory Tech.. vol. 45, pp. 519-525. Apr. 1997.

[4] P. Pelet and N. Engheta. "The theory of chirowaveguides", IEEE Trans. Antemas Propagat., vol. 38, pp. 90-98, Jan. 1990.

[5] L. Zhang, Y. Jiao, and C. Liang, "The dominant mode in a parallel-plate chirowaveguide". IEEE Trans. Microwave Theor Tech.. vol. 42, pp. 2009-2012. Oct. 1994.

[6] S. Maruyama, M. Koshiba. "Finite-element analysis of threedimensional chirowaveguides using hybrid edge/nodal elements", Electronics and Communications in Japan, Part 2 , vol. 78, no. 1 , pp. 6.3-72, 1995.

[7] J. Svedin, "Propagation analysis of chirowaveguides using the finite-element method", IEEE Trans. Microwave Theory Tech. vol. 38, no. 10. pp. 1889-1891. Oct. 1990.

[8] B. Katsenelenbaum, et a.l., "Chiral electromagnetic objects". Uspekhi Fizicheskikh Nauk. vol. 40. pp. 1149-1160, Nov. 1997.

[9] M. Norgren, Wave-Splitting Approaches to Direct and Inverse Frequency-Domain Scattering of Electromagnetic Waves from Stratified Bianisotropic Materials. Royal Institute of Technology, Department of Electromagnetic Theory, Stockholm. 1996.

Licinius Dimitri Sá de Alcantara nasceu em Belém. Brasilem 1975. Receheu o título de Tecnólogo em Processamento de Dados pelo Centro de Ensino Superior do Pará em 1995. Posteriormente, Recebeu os títulos de Engenheiro Eletricista em 1998 e de Mestre em Engenharia Elétrica em 1999 pela Universidade Federal do Pará. Atualmente ele está envolvido em projeto de doutorado na Universidade de São Paulo. Campus de São Carlos. SP.

Rubem Gonçalves Farias, nasceu em Afuá, PA. em 1950. Recebeu o título de Engenheiro Eletricista em 1974 pela ITFPA. Mestre em Engenharia Eletrônica pelo ITA. em $1983 \mathrm{e}^{\text {' }}$ Engenharia Elétrica em 1993 pela UNICAMP. At: Professor Titular em Eletromagnetismo no Departiun.... .. Engenharia Elétrica e de Computação da UFPA. Suas áreas de pesquisa incluem dispositivos para comunicações nas bandas ópticas, milimétrica e microondas. Dr. Rubem recebeu o Prêmio Volkswagen/UFPA pela conquista do primeiro lugar no Curso de Graduação do Centro Tecnológico da UFPA. versão 1974 